\title{
Relationship between pulmonary matrix metalloproteinases and quantitative CT markers of small airways disease and emphysema in COPD
}

\author{
Kristoffer Ostridge, ${ }^{1,2}$ Nicholas Williams, ${ }^{1,2}$ Viktoriya Kim, ${ }^{1,2}$ Michael Bennett, ${ }^{1}$ \\ Stephen Harden, ${ }^{3}$ Lindsay Welch, ${ }^{1}$ Simon Bourne, ${ }^{2}$ Ngaire A Coombs, ${ }^{4}$ \\ Paul T Elkington, ${ }^{1,2}$ Karl J Staples, ${ }^{2,5}$ Tom MA Wilkinson ${ }^{1,2,5}$
}

\begin{abstract}
- Additional material is published online only. To view, please visit the journal online (http://dx.doi.org/10.1136/ thoraxjn-2015-207428)
\end{abstract}

For numbered affiliations see end of article.

\section{Correspondence to} Dr Tom Wilkinson, Clinical and Experimental Sciences, University of Southampton Faculty of Medicine, Sir Henry Wellcome Laboratories, Southampton General Hospital, Mailpoint 810, Tremona Road, Southampton S016 6YD, UK: t.wilkinson@soton.ac.uk

Received 15 June 2015 Revised 2 November 2015 Accepted 9 November 2015 Published Online First 8 December 2015

\section{SLinked}

- http://dx.doi.org/10.1136/ thoraxjnl-2015-208204

\section{CrossMark}

To cite: Ostridge $\mathrm{K}$ Williams $\mathrm{N}$, Kim V, et al. Thorax 2016:71:126-132.

\section{ABSTRACT}

Background Matrix metalloproteinases (MMPs) are proteolytic enzymes that can degrade the extracellular matrix and drive tissue remodelling, key processes in the pathogenesis of COPD. The development of small airway disease has been identified as a critical mechanism in the early development of airflow obstruction but the contribution of MMPs in human disease is poorly characterised.

Objectives We investigated the role of MMPs and inflammatory cytokines in the lung by quantifying levels and determining relationships with the key pathological components of COPD in patients and healthy controls.

Methods We analysed levels of MMPs and inflammatory cytokines in bronchoalveolar lavage from 24 COPD and 8 control subjects. Each subject underwent spirometry and high-resolution CT. Image analysis quantitatively assessed emphysema, bronchial wall thickening and small airways disease.

Results Multiple MMPs (MMP-1, $-2,-3,-8,-9$ and -10 ) and cytokines (interleukin (IL) 6 and IL-8) were elevated in lungs of subjects with COPD. MMP-3, $-7,-8$, $-9,-10$ and -12 concentrations closely associated with CT markers of small airways disease. Emphysema severity was also associated with MMP-3, -7 and -10 . However, there were no strong relationships between MMPs and bronchial wall thickness of the larger airways.

Conclusions Pulmonary MMP concentrations are directly associated with the extent of gas trapping and small airways disease identified on CT scan. This study suggests that MMPs play a significant role in small airways remodelling, a key feature in the pathogenesis of COPD.

Trial registration number NCT01701869

\section{INTRODUCTION}

COPD is a heterogeneous disease characterised by the progressive development of airflow limitation which leads to functional impairment and associated symptoms. ${ }^{1}$ The underlying mechanisms of disease are poorly understood, which has limited the development of new therapeutic and diagnostic approaches. ${ }^{2}$

Tissue destruction, inflammation and airway remodelling are important features in COPD, and a number of pathways and mediators have been implicated in these processes. $^{3}$ A proteinase/

\section{Key messages}

What is the key question?

- Are matrix metalloproteinases (MMPs) involved in the development of pathological changes in the lung of patients with COPD and what is their association with emphysema formation bronchial wall thickening and small airways remodelling?

What is the bottom line?

- Bronchoalveolar lavage concentrations of multiple MMPs are increased in patients with COPD, and these are associated with the degree of small airways disease and emphysema measured by $\mathrm{CT}$ analysis.

\section{Why read on?}

- This study combines quantitative $\mathrm{CT}$ analysis with multiplex profiling of MMPs and inflammatory mediators to identify a new role of proteases in COPD.

antiproteinase imbalance has been postulated to be a key contributor to emphysematous changes. ${ }^{4}$ Matrix metalloproteinases (MMPs), a large family of zinc-dependent proteolytic enzymes, have the ability to degrade the pulmonary extracellular matrix (ECM) and have been implicated in COPD. ${ }^{4}$ They are broadly grouped depending on their substrate specificity, including collagenases (MMP-1, -8 and -13), gelatinases (MMP-2 and -9), stromelysins (MMP-3, -10 and -11) and elastases (MMP-7 and -12), ${ }^{5}$ although there is considerable substrate overlap. MMPs are not normally expressed in healthy tissue but in disease can be produced by alveolar macrophages, neutrophils and bronchial epithelial cells. ${ }^{5}$ They are tightly regulated by specific endogenous inhibitors, the four Tissue inhibitors of MMPs (TIMP). ${ }^{5}$

Transgenic mice over expressing MMP-1 develop emphysema at an accelerated rate. ${ }^{6}$ Selective inhibition of MMP-9 and -12 in guinea pigs reduced the extent of emphysema following exposure to smoke. ${ }^{7}$ Human studies demonstrate increased expression of MMP- $1,{ }^{8} \quad 9 \quad-8,,^{9} \quad 10 \quad-9^{8-13}$ and 
$-12^{8} 1415$ in the sputum or bronchoalveolar lavage (BAL) of subjects with COPD. However, detailed analysis of the MMP profile has not been integrated with systematic characterisation of lung pathology by high-resolution CT and lung function.

MMPs have complex biology with multistep activation and numerous molecular interactions, ${ }^{16}$ and the most important MMPs have yet to be determined in COPD. In addition, MMPs have multiple other functions that are independent of their ability to degrade the ECM, including facilitating cell migration and activating growth factors and cytokines. ${ }^{5}$ Most studies investigating MMPs in COPD have only investigated a subset of MMPs. Given their biological complexity, novel multiplex-based arrays provide the opportunity to profile a broad spectrum of MMPs in a carefully characterised clinical cohort.

The key sites of MMP activity in the lung are unknown and it is likely that matrix turnover and remodelling differs between regions. CT imaging provides the opportunity to study key morphological features of COPD including emphysema, bronchial wall morphology and small airways disease. Quantitative analysis software allows numerical, objective estimation of these disease facets. ${ }^{17}$ MMP-9 and -12 have been shown to have associations with quantitative measures of emphysematous change on $\mathrm{CT}^{11}{ }^{14}$ Previous studies have not investigated the association between MMP concentrations and bronchial wall measures or small airways disease identified by CT. In this study, we combine CT analysis of lung pathology with multiplex profiling of MMPs and inflammatory mediators, and identify a novel role for MMPs in COPD.

\section{METHODS}

Subjects

Subjects gave written informed consent and the study (ClinicalTrials.gov:NCT01701869) was approved by the South Central-Southampton B NRES Committee (12/SC/0304).

Twenty-four subjects with stable mild and moderate COPD as defined by GOLD guidelines ${ }^{1}$ were recruited into the study. Postbronchodilator spirometry was used to assess airflow obstruction with an FEV1:FVC ratio of $<0.7$ and an FEV1 of $\geq 50 \%$ predicted value required for enrolment. Spirometry was conducted in accordance with American Thoracic Society standards. ${ }^{18}$ Subjects had at least a smoking history of 10 pack years. Exclusion criteria included a history of other pulmonary disease, $\alpha$-1-antitrypsin deficiency, long-term antibiotics/steroids or an exacerbation within the month prior to recruitment. A control group of eight current or ex-smokers, with at least a 10 pack year history but preserved lung function were also recruited.

\section{CT scanning and quantitative image analysis}

Subjects underwent volumetric CT scans of the chest using a Siemens Sensation 64 CT scanner. The imaging protocol consisted of; slice thickness $0.75 \mathrm{~mm}$, slice separation $0.5 \mathrm{~mm}$, tube voltage $120 \mathrm{KV}$, effective mAs $90 \mathrm{mAs}$ (using dose modulation), collimation $0.6 \mathrm{~mm}$ and a pitch of 1 . Subjects were scanned at full inspiration. A subcohort of 22 subjects was scanned at endtidal volume. The remaining did not have an expiratory scan due to concerns over cumulative radiation exposure having had recent CT scans.

Images reconstructed with the B35 kernel were used for image analysis using Apollo Software (VIDA Diagnostics). Emphysema was quantified by the per cent of lung voxels on the inspiratory scan with attenuation values below $-950 \mathrm{HU}(\%$ LAA). Bronchial wall thickening was quantified using the standardised parameter Pi10, which is the square root of the wall area of a hypothetical airway with a $10 \mathrm{~mm}$ internal perimeter. A surrogate marker for small airways disease was measured using the ratio of mean lung attenuation on expiratory and inspiratory scans (E/I MLD), which has been previously validated. $^{19}$

\section{Bronchoscopy and sample acquisition}

Fibre optic bronchoscopy was performed on an outpatient basis. In each subject, two lobes were targeted, and BAL was performed by instilling $100 \mathrm{~mL}$ of $0.9 \%$ saline into each lobe and recovered by aspiration. BAL fluid was poured through $100 \mu \mathrm{m}$ filters and cells removed by $400 \mathrm{~g}$ centrifugation for $10 \mathrm{~min}$ at $4^{\circ} \mathrm{C}$. The supernatant was aliquoted and stored at $-80^{\circ} \mathrm{C}$ prior to analysis. The resulting cell pellets were prepared for cytospin analysis as previously described. ${ }^{20}$

\section{MMP and cytokine analysis}

MMP and cytokine concentrations in BAL were quantitated using a microparticle-based multiplex immunoassay (R\&D systems, Abingdon, UK) developed by Luminex. Samples were analysed on the Luminex 200 platform (Biorad Bioplex 200, Hemel Hempstead, UK), as per manufacturer's instructions. The following MMPs were analysed: MMP-1, -2, -3, -7, -8, -9, $-10,-12,-13$ and ECM metalloproteinase inducer (EMMPRIN), a cell surface molecule that can be shed. TIMP 1-4 were analysed and cytokine analysis was performed for interleukin (IL) $1 \beta$, IL-2, IL-6, IL-8, IL-10, GM-CSF, IFN $\gamma$ and TNF $\alpha$ (see online supplementary data for assay sensitivities).

\section{Statistical analysis}

Statistical analyses were performed using SPSS V.21. MannWhitney U test and Fisher's exact tests compared data between COPD and control groups. In the subjects with COPD, associations between MMPs, spirometry, CT parameters and cytokines were assessed using Spearman's correlation with $r$ and $p$ values presented. Partial Spearman's correlation was used to conduct multivariate analysis. Each subject had two lobes sampled, and the mean concentrations between the lobes were used. For the purpose of statistical analysis, values that were below the lower limit of detection were given the value of half the concentration of detection. A p value of $<0.05$ was considered statistically significant.

Table 1 Characteristics of participants included in the study

\begin{tabular}{lllc}
\hline & COPD $(\mathbf{n}=24)$ & Controls $(\mathbf{n}=8)$ & $\mathbf{p}$ Value \\
\hline Age & $66.0(12.0)$ & $56.0(18.0)$ & 0.064 \\
Male & 16 & 6 & $>0.999$ \\
Current smoker & 11 & 5 & 0.685 \\
FEV1\% & $69.00(21.00)$ & $108.00(20.25)$ & $<0.001^{*}$ \\
FEV1/FVC & $54.50(10.75)$ & $78.00(9.50)$ & $<0.001^{*}$ \\
FEF25\%-75\% & $21.50(16.0)$ & $77.50(45.25)$ & $<0.001^{*}$ \\
Emphysema \% (\%LAA) & $8.24(7.17)$ & $2.55(3.35)$ & $0.029^{*}$ \\
Bronchial wall area (Pi10) & $3.74(0.15)$ & $3.74(0.04)$ & 0.502 \\
Small airways disease (E/I MLD) & $0.86(0.08)$ & $0.76(0.10)$ & $0.002^{*}$
\end{tabular}

Values are given as medians (IQR).

Male and current smokers given as number of subjects.

$\%$ LAA and Pi10 given for 31 subjects. The ratio of mean lung density in expiration to inspiration (E/I MLD) is given for a subcohort of 22 subjects who had expiratory CT scans.

Fisher's exact test for male and current smoker. Mann-Whitney $U$ test for all other variables.

${ }^{*} \mathrm{p}<0.05$ 


\section{RESULTS}

Subject characteristics

COPD and control groups were well matched for age, sex and current smoking status (table 1). There were more males in both groups. FEV1\% and FEF25\%-75\% were significantly lower in the COPD group.

\section{MMP concentrations in COPD and controls}

Median MMP-1, -2, -3, -8, -9 and -10 were significantly higher in the BAL of subjects with COPD compared with controls (figure 1). There was no significant difference in MMP-7, -12, -13 and EMMPRIN between the groups.

\section{Neutrophil and cytokine concentrations in COPD and} controls

BAL neutrophil count, IL-6 and IL-8 concentrations were significantly higher in subjects with COPD than controls (figure 2).
There were no significant differences in IL-1 $\beta$ and GM-CSF between groups. IL-2, IL-10, IFN $\gamma$ and TNF- $\alpha$ were only detected at very low concentration in all samples and so further analysis was not performed.

\section{Associations between MMPs, cytokines, lung function and CT parameters}

We investigated the relationships between MMPs, cytokines and physiological and CT parameters in the subjects with COPD.

\section{Relationships of MMPs with cytokines and neutrophils}

We performed a systematic analysis of associations between cytokines, neutrophils and tissue-destructive MMPs, using Spearman's correlation. There were significant associations between the cytokines IL- 6 and IL- 8 and MMP-1, -7, -8, -9, -12 and EMMPRIN (table 2). GM-CSF was associated with MMP-8, -9 and EMMPRIN. IL-1 $\beta$ had a negative correlation
A

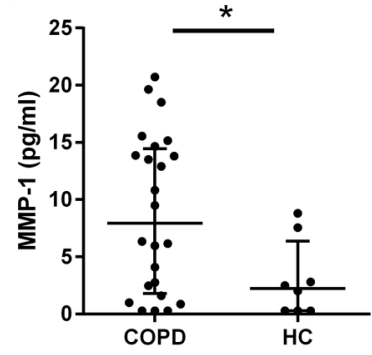

D

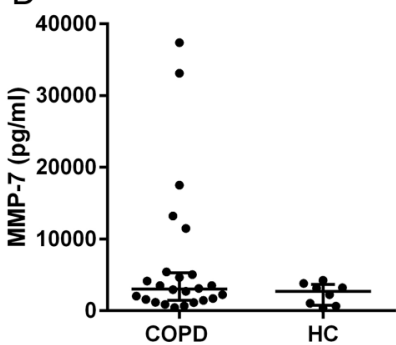

G

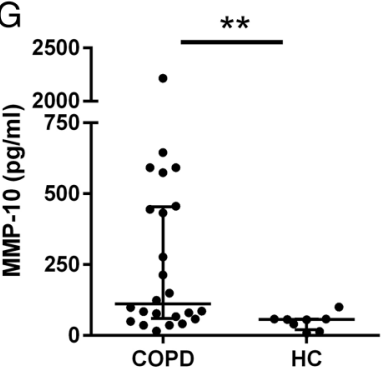

$J$

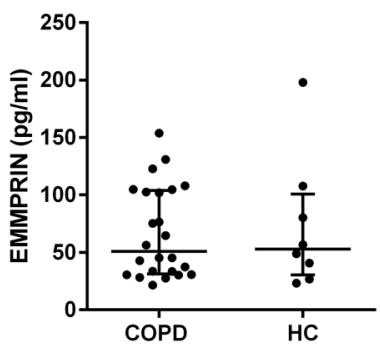

B

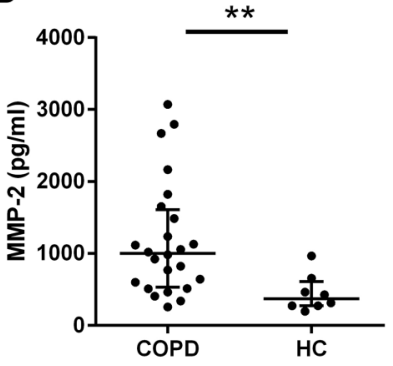

E

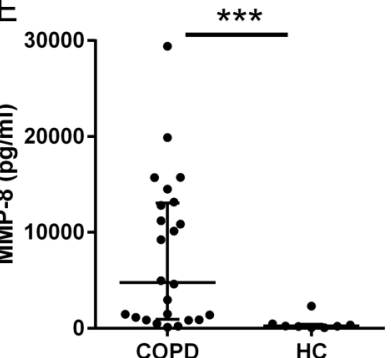

$\mathrm{H}$

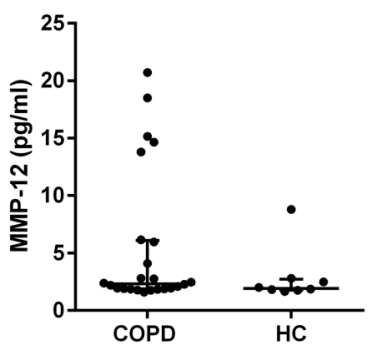

C

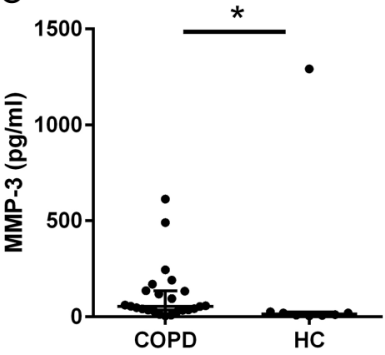

$\mathrm{F}$

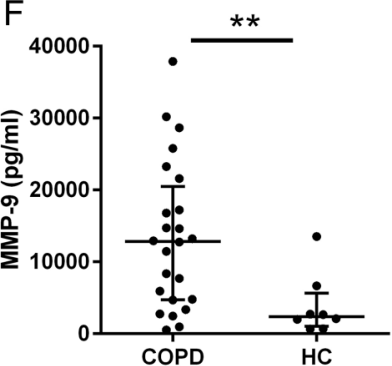

I

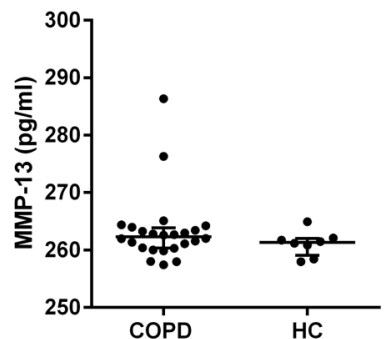

Figure 1 Bronchoalveolar lavage (BAL) expression of matrix metalloproteinases (MMPs) in COPD and controls (HC). (A) MMP-1, (B) MMP-2, (C) MMP-3, (D) MMP-7, (E) MMP-8, (F) MMP-9, (G) MMP-10, (H) MMP-12, (I) MMP-13, (J) extracellular matrix metalloproteinase inducer (EMMPRIN). Data represents median with IQR. Each dot represents BAL concentration of individual MMP in a specific patient, $n=24$ for COPD and 8 for controls. ${ }^{*} \mathrm{p}<0.05,{ }^{* *} \mathrm{p}<0.01,{ }^{* * *} \mathrm{p}<0.001$ using Mann-Whitney $U$ test. 
Figure 2 Bronchoalveolar lavage (BAL) neutrophils and cytokines in COPD and controls (HC). (A) differential BAL neutrophil count (\%), (B) IL-1 $\beta$, (C) IL-6, (D) IL-8, (E) GM-CSF. Data represent median with IQR. Each dot represents BAL concentration of individual value in a specific patient, $n=24$ for COPD and 8 for controls. ${ }^{*} p<0.05,{ }^{* *} p<0.01$ using Mann-Whitney U test. IL, interleukin.
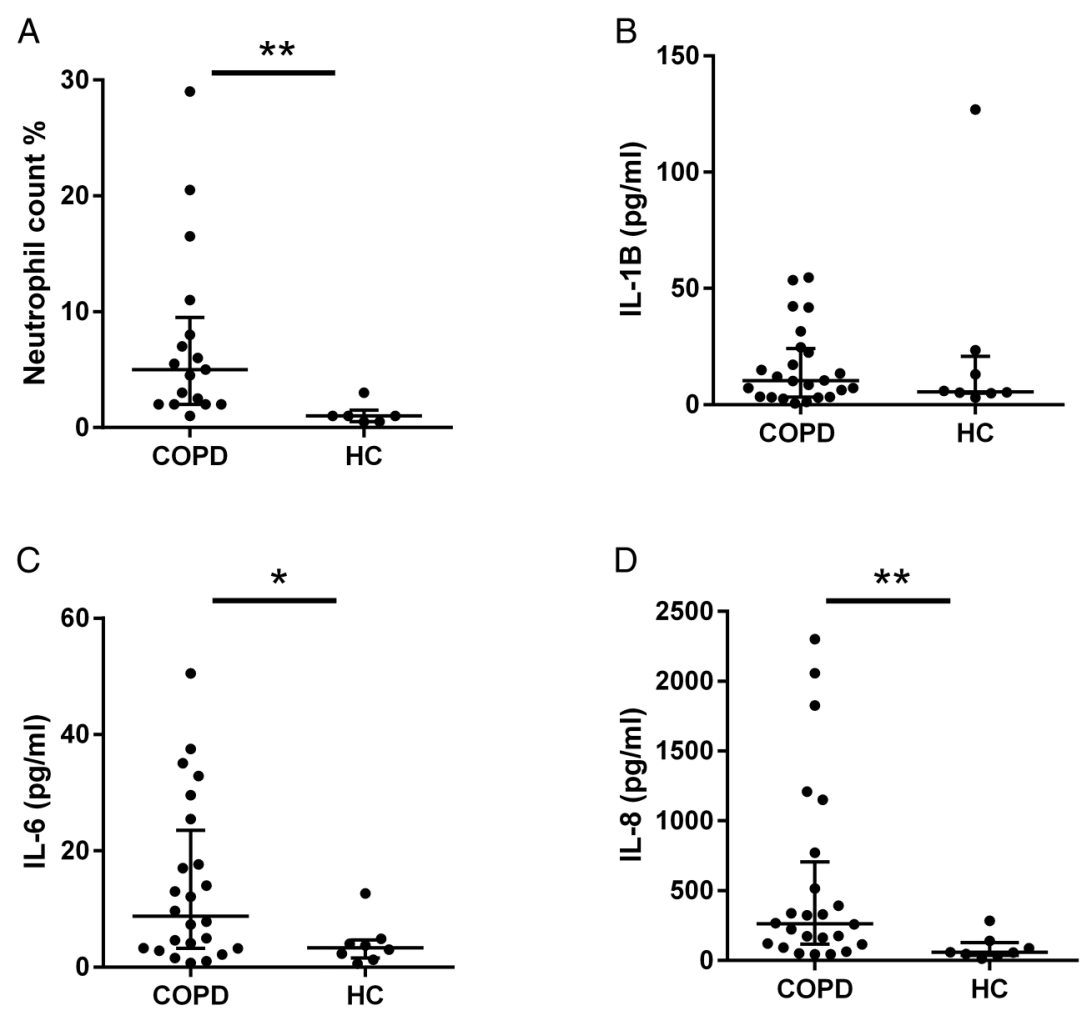

E

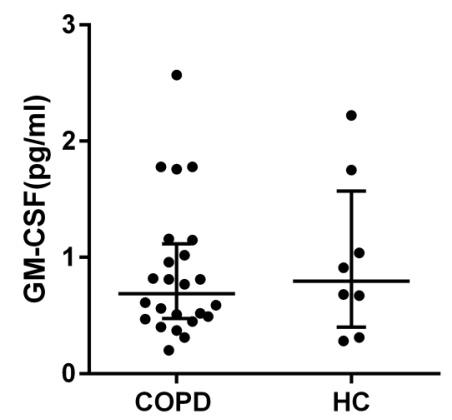

with MMP-3 while neutrophil counts were not associated with any MMPs.

\section{Relationships of MMPs, cytokines, neutrophils with lung function}

We compared MMP and cytokine concentrations with lung function markers of disease severity using Spearman's correlation. There were significant associations between airflow obstruction (FEV1\%) and MMP-8, -9 and -12 and between FEF25\%-75\% and MMP-1, -8, -9 and -12 (table 2). MMP-8 had the strongest association with both FEV1\% and FEF25\%$75 \%$ (figure 3) and using partial correlation this remained significant when adjusting for each of the other MMPs in turn apart from MMP-9 (see online supplementary table S1).

FEV1\% and FEF25\%-75\% were significantly associated with IL-8. FEF25\%-75\% was also associated with IL-1 $\beta$. There was no association between these spirometric markers and BAL neutrophils, IL-6 and GM-CSF (see online supplementary table S2).

\section{CT analysis}

Quantitative CT analysis was performed to study emphysema, bronchial wall morphology and small airways disease and assess the relationships with MMPs and cytokines. Segmentation and quantitative analysis was successfully achieved in 31 subjects. One of the control CT scans could not be analysed for technical reasons.

\section{Emphysema}

Subjects with COPD had significantly more emphysema (higher \%LAA) than controls (table 1), although there was no significant association between emphysema and FEV1\% in the subjects with COPD ( $\mathrm{r}-0.10, \mathrm{p}=0.63)$.

There were significant associations between emphysema ( $\%$ LAA) and MMP-3, -7 and -10 (table 2). MMP-10 had the strongest association with emphysema (figure 3) and using partial correlation this remained significant when adjusting for each of the other MMPs apart from MMP-3 and -7 (see online supplementary table S1). Emphysema was not associated with neutrophils or cytokines (see online supplementary table S2).

\section{Small airways disease}

Small airways disease (E/I MLD) was greater in the COPD group than controls (table 1). Strong associations with FEV1\% $(\mathrm{r}-0.72, \mathrm{p}=0.002)$ and FEF25\%-75\% $(\mathrm{r}-0.54, \mathrm{p}=0.03)$ were seen in subjects with COPD. 
Table 2 Spearman's correlation analysis between matrix metalloproteinases (MMPs), spirometry, CT measures of disease and cytokines and neutrophils in subjects with COPD

\begin{tabular}{|c|c|c|c|c|c|c|c|c|c|c|}
\hline & MMP-1 & MMP-2 & MMP-3 & MMP-7 & MMP-8 & MMP-9 & MMP-10 & MMP-12 & MMP-13 & EMMPRIN \\
\hline \multicolumn{11}{|l|}{ Cytokines and neutrophils } \\
\hline IL-1 $\beta$ & 0.26 & -0.20 & $-0.44^{*}$ & 0.08 & 0.30 & 0.21 & -0.21 & -0.00 & 0.18 & 0.22 \\
\hline IL-6 & $0.51^{*}$ & -0.18 & 0.28 & $0.55^{* *}$ & $0.69^{* * *}$ & $0.55^{* *}$ & 0.21 & $0.52^{* *}$ & -0.22 & $0.54^{* *}$ \\
\hline IL-8 & $0.44^{*}$ & -0.26 & 0.06 & $0.53^{* *}$ & $0.90^{* * *}$ & $0.78^{* * *}$ & 0.16 & $0.56^{* *}$ & -0.01 & $0.46^{*}$ \\
\hline GM-CSF & -0.02 & -0.16 & -0.25 & 0.15 & $0.41^{*}$ & $0.41^{*}$ & 0.13 & 0.20 & -0.31 & $0.68 * * *$ \\
\hline Neutrophils & -0.12 & 0.07 & 0.44 & 0.40 & 0.41 & 0.28 & 0.25 & 0.29 & -0.26 & 0.36 \\
\hline \multicolumn{11}{|l|}{ Spirometry } \\
\hline FEV1\% & -0.35 & 0.28 & -0.34 & -0.35 & $-0.60^{* *}$ & $-0.59^{* *}$ & -0.19 & $-0.51^{*}$ & 0.02 & -0.20 \\
\hline FEF $25 \%-75 \%$ & $-0.47^{*}$ & 0.39 & -0.03 & -0.18 & $-0.61 * *$ & $-0.58^{* *}$ & -0.11 & $-0.44^{*}$ & 0.22 & -0.22 \\
\hline \multicolumn{11}{|l|}{ CT parameters } \\
\hline Emphysema \% (LAA\%) & -0.03 & -0.05 & $0.49^{*}$ & $0.52^{* *}$ & 0.35 & 0.33 & $0.52^{*}$ & 0.09 & -0.24 & 0.14 \\
\hline Small airways disease (E/I MLD) & 0.16 & -0.14 & $0.52^{*}$ & $0.52^{*}$ & $0.60 *$ & $0.56^{*}$ & $0.55^{*}$ & $0.50^{*}$ & -0.03 & 0.32 \\
\hline Bronchial wall area (Pi10) & 0.12 & 0.08 & 0.04 & 0.11 & 0.23 & 0.21 & 0.13 & 0.17 & 0.20 & 0.15 \\
\hline $\begin{array}{l}\text { Spearman's } r \text { values given. } \\
N=24, \text { apart from associations with } \\
{ }^{*} p<0.05,{ }^{* *} p<0.01,{ }^{* * *} p<0.001 \text {. } \\
\text { IL, interleukin. }\end{array}$ & $n-$ & & & & & & & & & \\
\hline
\end{tabular}

There were associations between small airways disease (E/I MLD) and MMP-3, -7, -8, -9, -10 and -12 (table 2). MMP-8 had the strongest association with small airways disease (figure 3 ) and this remained significant after adjusting for MMP-1, -2 and -13 in turn but not the other MMPs (see online supplementary table S1). The only cytokine that correlated with small airways disease was IL-8 (see online supplementary table S2).

\section{Bronchial wall thickening}

There was no significant difference in bronchial wall thickening (Pi10) between COPD and controls, but there was an association between Pi10 and FEV1\% in subjects with COPD (r $-0.59, \mathrm{p}=0.003)$. There were no associations between airway wall area and MMPs (table 2). Neutrophils and cytokines did not show any association with bronchial wall area (see online supplementary table S2).

\section{DISCUSSION}

This study has, for the first time, demonstrated an association between small airways disease measured by CT imaging and the lung concentrations of MMPs and inflammatory cytokines in COPD. MMPs and cytokines are also associated with other markers of disease severity, including FEV1\% and emphysema severity. The analysis suggests that associations were stronger between MMPs and small airways disease rather than emphysema.

MMPs are structurally similar proteolytic enzymes that have been implicated in the tissue remodelling, matrix destruction
Figure 3 Scatter plots of subjects with COPD for (A) log matrix metalloproteinase (MMP)-8 against FEV1\%, (B) log MMP-8 against log FEF25\%-75\%, (C) log MMP-10 against LAA\%, (D) log MMP-8 against E/I MLD. These plots are visualised as they represent the MMPs with the strongest associations with the accompanying outcome variable. $\mathrm{N}=24$, apart from E/l MLD $(n=16)$.
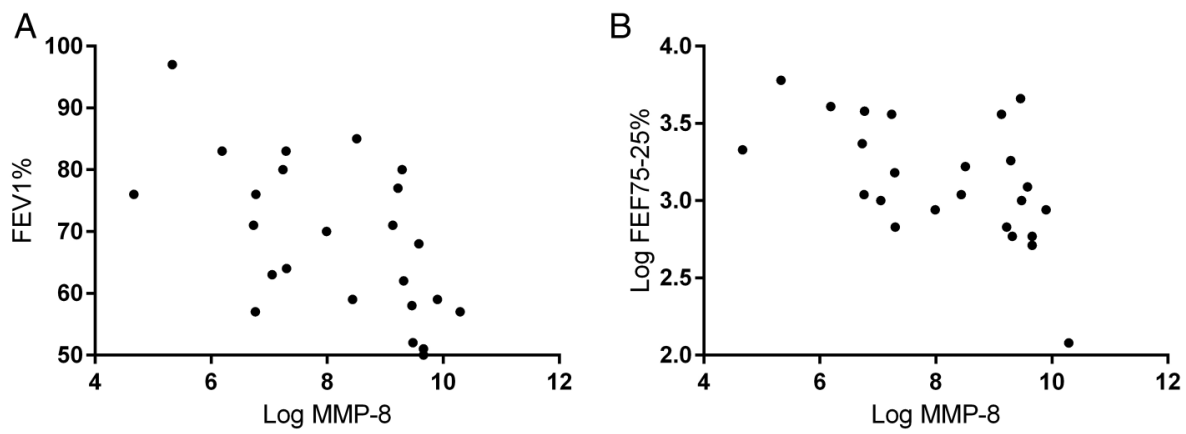

C

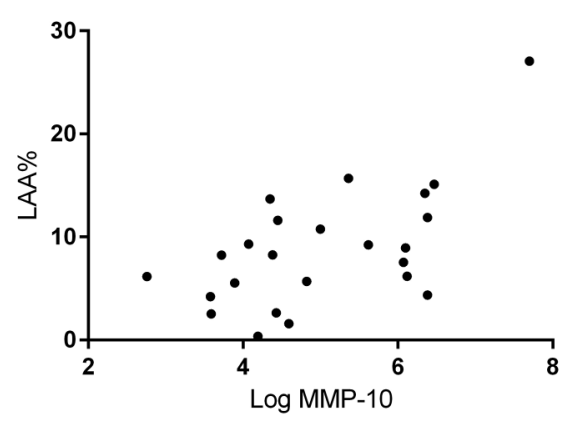

D

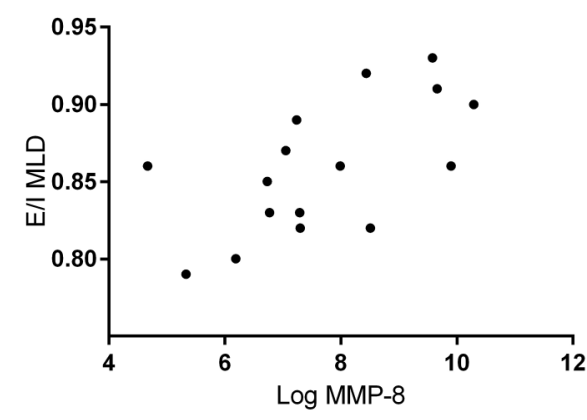


and inflammation seen in COPD. ${ }^{4}$ We found that MMP-1, -2, $-3,-8,-9$ and -10 were elevated in the airways of subjects with COPD. This is in keeping with previous studies which demonstrated that MMP-1, ${ }^{8} 9-8^{9} 10$ and $-9^{8-13}$ are increased in subjects with COPD. Furthermore, we have also shown that MMP-2, -3 and -10 were increased. A histological study of lung resection subjects also found that MMP-2 was upregulated in the lung periphery in COPD. ${ }^{21}$ However, Culpitt et al ${ }^{9}$ found no difference between MMP-2 and -3 in sputum between COPD and controls. Our results may diverge from these due to the difference in sampling locations between sputum and BAL. Previous studies also found that MMP-12 is increased in COPD ${ }^{8} 1415$ although we did not replicate this observation in our cohort. We analysed the ratio of MMPs: TIMPs and found they were significantly increased in subjects with COPD (data not shown) and this was especially the case for MMP-8 and -10. This supports the idea of a proteinase to antiproteinase imbalance; however, further work is required to understand this fully. We also found the concentrations of neutrophils, IL-6 and IL-8 were increased in COPD, which is consistent with previous work. $^{22}$ This provides further evidence for the role of neutrophilic airway inflammation in COPD.

Most work to date has focused on assessing the role of MMPs in emphysema. Transgenic mice overexpressing MMP-1 develop emphysema at an accelerated rate. ${ }^{6}$ MMP-12 knockout mice are protected from cigarette induced lung damage. ${ }^{23}$ In the SMAD3 mouse model of emphysema, genetic blockade of MMP-9 reduced the amount of emphysema. ${ }^{24}$ Selective inhibition of MMP-9 and -12 in guinea pigs reduced the extent of emphysema when exposed to smoke. ${ }^{7}$ In human genetic studies, polymorphisms of MMP-9 genes have been linked to emphysematous change $\mathrm{e}^{25} 26$ while polymorphisms of MMP-12 have been linked to airflow obstruction. ${ }^{27}$ Previous work using lavage fluid found that MMP-1, -9 and -12 were significantly raised in patients with emphysema when compared with healthy nonsmokers but not current smokers. ${ }^{8}$ In two studies using quantitative CT analysis, the LAA\% correlated with sputum MMP-9 and -12. ${ }^{11}{ }^{14}$ Unlike these two studies, we did not find any associations between CT-measured emphysema and MMP-9 or -12, which may be due to the differences between sampling sputum or BAL. However, we found significant associations between quantified emphysema and MMP-3, -7 and -10, which have not been previously investigated. MMP-10 had the strongest association with emphysema, and interestingly, this and the other significantly associated MMPs have limited elastin degradation properties and mainly degrade collagens and proteoglycans. Studies show that there is actually increased collagen around emphysematous lesions, ${ }^{28}$ and therefore a key mechanism may be MMP-induced collagen breakdown and subsequent abnormal remodelling and aberrant deposition of collagen.

We measured bronchial wall thickening of larger airways using the standardised marker Pi10 which has been shown to correlate with FEV1\% $\%^{29}$ and is elevated in COPD. ${ }^{30}$ Our analysis found no significant difference in Pi10 between COPD and controls but did show an association with airflow obstruction. There were no significant associations between Pi10 and MMPs or inflammatory cytokines. The reasons for this are unknown, but it may be that Pi10 is not the best measure at assessing airway wall morphology. It is also feasible that bronchial wall thickening is not directly linked to luminal inflammatory indices or protease activity and the remodelling response in the proximal airway may be more related to inflammatory infiltration of the submucosa ${ }^{31}$ and biopsy studies may be required to elicit these mechanisms.
Small airways disease is a key feature of COPD and has been identified as the main site of the airflow obstruction seen in the disease. $^{32}$ Histopathological studies have identified that the small airways are thickened and morphologically abnormal with a combination of squamous cell metaplasia, goblet cell hyperplasia and peribronchial fibrosis apparent. ${ }^{33}$ There is currently no gold standard for investigating and assessing small airways disease in COPD. CT lacks the resolution to image the small airways directly; however, the indirect sign of gas trapping can be used. ${ }^{17} \mathrm{~A}$ number of quantitative CT methods exist to measure this, and we chose to use the ratio of mean lung density in expiration against inspiration. Hersh et $a l^{34}$ confirmed that E/I MLD had a good correlation with lung function markers of small airways disease. Bommart also demonstrated that E/I MLD was the best CT marker of gas trapping as measured by nitrogen washout. ${ }^{19}$ We found E/I MLD was significantly raised in COPD and had strong associations with FEV1\% and FEF25\%-75\%, supporting its use as a marker of small airway disease.

Few studies have investigated the role of MMPs in small airways disease. In guinea pigs, inhibition of MMP-9 and -12 protected the animals from small airways fibrosis in response to cigarette smoke exposure. ${ }^{7}$ A study of symptomatic smokers with normal lung function found that MMP-8, -9 and -12 in induced sputum correlated with FEF25\%-75\%. ${ }^{35}$ Our results found that MMP-1, $-8,-9$ and -12 had associations with FEF25\%-75\%. However this is an insensitive and non-specific marker of small airways disease. When using the CT marker E/I MLD we found strong associations with the concentrations of MMP-3, -7, -8, -9, -10 and -12. These associations tended to be stronger than those seen with emphysema, suggesting that MMPs may play a significant role in the development of small airway remodelling and the associated airflow obstruction. MMP-8 had the strongest association with small airways disease and along with the other associated MMPs is mainly produced by neutrophils and macrophages and between them are able to degrade all components of the ECM. In the small airways, it has been demonstrated that elastin and collagen are reduced in patients with COPD, ${ }^{36}$ and we propose that this degradation is driven by MMPs leading to deranged remodelling and fibrosis.

We recognise that the main limitation of this study was the small sample size and associated limited statistical power, meaning that we were unable to analyse certain aetiological factors such as smoking. However, every effort was made to phenotype our cohort with CT imaging and invasive bronchoscopy techniques. Despite this, we found strong evidence of the associations between MMPs and clinical features of disease. Another limitation is the multiple comparisons made in this study. We tested 129 associations between parameters of physiology, CT indices and laboratory markers. At the 5\% level, around seven associations would be expected to be significant just by chance. We found 38 significant associations, far higher than the number expected by chance, suggesting the presence of genuine associations. Due to the need to perform bronchoscopy, our study consisted of patients with mild and moderate COPD, with only limited amounts of emphysema. It is unknown whether these results would be the same in more severe patients. In this study, we measured concentrations of MMPs rather than MMP activity. Lowrey demonstrated that although MMP-9 concentrations were increased in the sputum of subjects with COPD, MMP-9 activity was not. ${ }^{12}$ However, due to the site and nature of action of MMPs, there is considerable debate as to whether concentrations or activity assays best reflect the action of MMPs. ${ }^{37}$ Another limitation was that only a subcohort 
of 16 subjects with COPD had both an inspiratory and expiratory CT scan, allowing assessment of small airways disease. Repeating emphysema and airflow analysis on this subsample yielded broadly similar results, indicating the smaller sample size did not influence the results.

In conclusion, multiple MMPs are increased in the airways of subjects with COPD and are associated with the severity of airflow obstruction and quantitative CT measures of emphysema and small airways disease. This suggests that MMPs may play a significant role in the pathogenesis of COPD by causing breakdown of the pulmonary ECM leading to abnormal remodelling in both the small airways and lung parenchyma. Further work is required to investigate these important mechanisms and to understand the heterogeneity of the disease within different compartments of the lung. While most previous work has focused on MMPs and emphysema, this study suggests the strongest associations were with small airways disease. Interventions directed at inhibiting MMPs may have a role in preventing small airways remodelling, and any trial investigating modulation of MMPs should use CT analysis as a marker of small airways disease.

\author{
Author affiliations \\ 'Southampton NIHR Respiratory Biomedical Research Unit, Southampton General \\ Hospital, Southampton, UK \\ ${ }^{2}$ Department of Clinical \& Experimental Sciences, University of Southampton Faculty \\ of Medicine, Southampton General Hospital, Southampton, UK \\ ${ }^{3}$ Department of Radiology, University Hospital Southampton, Southampton General \\ Hospital, Southampton, UK \\ ${ }^{4}$ Department of Primary Care \& Population Sciences, University of Southampton \\ Faculty of Medicine, Southampton General Hospital, Southampton, UK \\ ${ }^{5}$ Wessex Investigational Sciences Hub, University of Southampton Faculty of \\ Medicine, Southampton General Hospital, Southampton, UK
}

Twitter Follow Simon Bourne at @COPDeducation

Acknowledgements We would like to express our appreciation to the staff of the Southampton NIHR Respiratory Biomedical Research Unit, in particular Kate Lippiett, Shuna Egerton, Sukh Purewal and Sarah Bawden. We also acknowledge the important contributions of the GSK vaccine team who provided funding and project management. We extend our gratitude to all the volunteers who gave their time and enthusiasm to make this research possible.

Contributors Conception and design: KO, LW, PTE, KJS and TMAW; data acquisition, analysis and interpretation: all authors; drafting of manuscript for important intellectual content: KO, KJS and TMAW.

Funding This study was funded by GSK Biologicals, Belgium via a Collaborative Research and Development Agreement (CRADA). No restrictions were placed on authors regarding the statements made in the manuscript.

Competing interests None declared.

Ethics approval NRES Committee South Central—Southampton B (12/SC/0304).

Provenance and peer review Not commissioned; externally peer reviewed.

\section{REFERENCES}

1 Rabe KF, Hurd S, Anzueto A, et al. Global strategy for the diagnosis, management, and prevention of chronic obstructive pulmonary disease: GOLD executive summary. Am J Respir Crit Care Med 2007;176:532-55.

2 Barnes PJ. Emerging pharmacotherapies for COPD. Chest 2008;134:1278-86.

3 Fischer BM, Pavlisko E, Voynow J. Pathogenic triad in COPD: oxidative stress, protease-antiprotease imbalance, and inflammation. Int I Chron Obstruct Pulmon Dis 2011;6:413-21.

4 Churg A, Zhou S, Wright JL. Series 'matrix metalloproteinases in lung health and disease': matrix metalloproteinases in COPD. Eur Respir J 2012;39:197-209.

5 Elkington PTG, Friedland JS. Matrix metalloproteinases in destructive pulmonary pathology. Thorax 2006;61:259-66.

6 Foronjy RF, Okada Y, Cole R, et al. Progressive adult-onset emphysema in transgenic mice expressing human MMP-1 in the lung. Am J Physiol Lung Cell Mol Physiol 2003;284:L727-37.

7 Churg A, Wang R, Wang X, et al. Effect of an MMP-9/MMP-12 inhibitor on smoke-induced emphysema and airway remodelling in guinea pigs. Thorax 2007:62:706-13.
8 D'Armiento JM, Goldklang MP, Hardigan AA, et al. Increased matrix metalloproteinase (MMPs) levels do not predict disease severity or progression in emphysema. PLOS ONE 2013;8:e56352.

9 Culpitt SV, Rogers DF, Traves SL, et al. Sputum matrix metalloproteases: comparison between chronic obstructive pulmonary disease and asthma. Respir Med 2005;99:703-10.

10 Vernooy JHJ, Lindeman JHN, Jacobs JA, et al. Increased activity of matrix metalloproteinase- 8 and matrix metalloproteinase- 9 in induced sputum from patients with COPD. Chest 2004;126:1802-10.

11 Chaudhuri R, McSharry C, Spears M, et al. Sputum matrix metalloproteinase-9 is associated with the degree of emphysema on computed tomography in COPD. Trans/ Respir Med 2013;1:11.

12 Lowrey GE, Henderson N, Blakey JD, et al. MMP-9 protein level does not reflect overall MMP activity in the airways of patients with COPD. Respir Med 2008;102:845-51.

13 Beeh KM, Beier J, Kornmann 0, et al. Sputum matrix metalloproteinase-9, tissue inhibitor of metalloprotinease-1, and their molar ratio in patients with chronic obstructive pulmonary disease, idiopathic pulmonary fibrosis and healthy subjects Respir Med 2003;97:634-9.

14 Chaudhuri R, McSharry C, Brady J, et al. Sputum matrix metalloproteinase-12 in patients with chronic obstructive pulmonary disease and asthma: relationship to disease severity. J Allergy Clin Immunol 2012;129:655-63.

15 Demedts IK, Morel-Montero, A., Lebecque S, et al. Elevated MMP-12 protein levels in induced sputum from patients with COPD. Thorax 2006;61:196-201.

16 Greenlee KJ, Werb Z, Kheradmand F. Matrix metalloproteinases in lung: multiple, multifarious, and multifaceted. Physiol Rev 2007:87:69-98.

17 Hackx M, Bankier AA, Gevenois PA. Chronic obstructive pulmonary disease: CT quantification of airways disease. Radiology 2012:265:34-48.

18 Miller MR, Hankinson J, Brusasco V, et al. Standardisation of spirometry. Eur Respir J 2005;26:319-38.

19 Bommart S, Marin G, Bourdin A, et al. Relationship between CT air trapping criteria and lung function in small airway impairment quantification. BMC Pulm Med 2014;14:29.

20 Grainge C, Lau L, Ward J, et al. Effect of bronchoconstriction on airway remodeling in asthma. N Engl J Med 2011;364:2006-15.

21 Baraldo S, Bazzan E, Zanin ME, et al. Matrix metalloproteinase-2 protein in lung periphery is related to COPD progression. Chest 2007;132:1733-40.

22 Barnes PJ. The cytokine network in asthma and chronic obstructive pulmonary disease. J Clin Invest 2008:118:3546-56.

23 Hautamaki RD, Kobayashi DK, Senior RM, et al. Requirement for macrophage elastase for cigarette smoke-induced emphysema in mice. Science 1997:277:2002-4.

$24 \mathrm{Xu} \mathrm{B}, \mathrm{Chen} \mathrm{H}, \mathrm{Xu} \mathrm{W}$, et al. Molecular mechanisms of MMPg overexpression and its role in emphysema pathogenesis of Smad3-deficient mice. Am J Physiol Lung Cell Mol Physiol 2012;303:L89-96.

25 Kukkonen MK, Tiili E, Vehmas T, et al. Association of genes of protease-antiprotease balance pathway to lung function and emphysema subtypes. BMC Pulm Med 2013:13:36.

26 Ito I, Nagai S, Handa T, et al. Matrix metalloproteinase-9 promoter polymorphism associated with upper lung dominant emphysema. Am J Respir Crit Care Med 2005;172:1378-82.

27 Hunninghake GM, Cho MH, Tesfaigzi Y, et al. MMP12, lung function, and COPD in high-risk populations. N Engl J Med 2009;361:2599-608.

28 Finlay GA, Donnell MDO, Connor CMO, et al. Elastin and collagen remodeling in emphysema. A scanning electron microscopy study. Am J Pathol 1996;149:1405-15.

29 Martinez $\mathrm{CH}$, Chen Y-H, Westgate PM, et al. Relationship between quantitative CT metrics and health status and BODE in chronic obstructive pulmonary disease. Thorax 2012:67:399-406.

30 Rutten EPA, Grydeland TB, Pillai SG, et al. Quantitative CT: associations between emphysema, airway wall thickness and body composition in COPD. Pulm Med 2011:2011:419328.

31 O'Donnell R, Breen D, Wilson S, et al. Inflammatory cells in the airways in COPD. Thorax 2006:61:448-54.

32 Hogg JC, Macklem PT, Thurlbeck WM. Site and nature of airway obstruction in Chronic Obstructive lung disease. N Engl J Med 1968;278:1355-60.

33 Burgel P-R, Bourdin A, Chanez P, et al. Update on the roles of distal airways in COPD. Eur Respir Rev 2011;20:7-22.

34 Hersh CP, Washko GR, Estépar RSJ, et al. Paired inspiratory-expiratory chest CT scans to assess for small airways disease in COPD. Respir Res 2013:14:42.

35 Ilumets $H$, Rytilä $P$, Demedts I, et al. Matrix metalloproteinases $-8,-9$ and -12 in smokers and patients with stage 0 COPD. Int J Chron Obstruct Pulmon Dis 2007;2:369-79.

36 Annoni R, Lanças T, Yukimatsu Tanigawa $R$, et al. Extracellular matrix composition in COPD. Eur Respir J 2012:40:1362-73.

37 Murphy $\mathrm{G}$, Nagase $\mathrm{H}$. Localizing matrix metalloproteinase activities in the pericellular environment. FEBS J 2011:278:2-15. 